\title{
TABLE OF CONTENTS INHALTSVERZEICHNIS
}

ABOUT THE PROJECT / ÜBER DAS PROJEKT 6

ABOUT THE BOOK / ÜBER DAS BUCH

$\nearrow$ Gerald Bast

PREFACE / VORWORT

$\nearrow$ Christoph Kaltenbrunner

DO RESEARCH! / FORSCHEN!

$[1.0] \begin{aligned} & \text { PRECONDITIONS } \\ & \text { FRAGESTELLUNGEN }\end{aligned}$

[1.1] MATERIAL / MATERIAL 21

$\nearrow$ Helga Lichtenegger 22

CELLULOSE NANOFIBERS IN WOOD:

Tiny Structural Elements with a Huge Effect

ZELLULOSE-NANOFASERN IN HOLZ:

Kleine Strukturelemente mit großer Wirkung

$\nearrow$ Ulrich Müller, Alfred Teischinger

CONNECTIONS IN WOOD AND MATERIAL EFFICIENCY:

Wood Formation Follows Mechanical Load

VERBINDUNGEN IM HOLZ UND MATERIALEFFIZIENZ:

Die Holzbildung folgt der mechanischen Belastung

[1.2] CRAFT / HANDWERK 39

$\nearrow$ Klaus Zwerger

HANDICRAFT AND TECHNOLOGY

HANDWERK UND TECHNOLOGIE

$\nearrow$ Brigitte Felderer

HANDICRAFT AS AN ATTITUDE TOWARDS THE WORLD

HANDWERK ALS HALTUNG ZUR WELT

$[2.0]$ CONCEPTUAL JOINING

APPROACH / ANSATZ

[2.1] INTERLOCKING SPACES / INTERLOCKING SPACES 65

INTRO / EINLEITUNG 66

$\nearrow$ Jun Sato 68

DEVELOPMENT OF KIGUMI JOINERY TO CREATE 'NATURALNESS'

ENTWICKLUNG DER KIGUMI-TECHNIK ZUR SCHAFFUNG VON ,NATÜRLICHKEIT'

JOINTS / VERBINDUNGEN 76

$\nearrow$ Karin Raith 90

WOOD CONSTRUCTION - ON THE RENEWAL OF AN ANCIENT ART

HOLZBAU - ZUR ERNEUERUNG EINER ALTEN KUNST 
TOOLS / WERKZEUGE

$\nearrow$ Adam Orlinski, Clemens Preisinger, Moritz Heimrath

ENCODED STRUCTURES:

Constraint-Based Design of Modular Elements

CODIERTE STRUKTUREN:

Constraint-basierte Konstruktion modularer Elemente

GEOMETRY / GEOMETRIE

[2.2] BRANCH FORMATIONS / BRANCH FORMATIONS 129

INTRO / EINLEITUNG $\quad 130$

GEOMETRY / GEOMETRIE

$\nearrow$ Anja Jonkhans $\quad 152$

DIGITAL DESIGN IN RESEARCH AND PRACTICE

DIGITALES DESIGN IN FORSCHUNG UND PRAXIS

TOOLS / WERKZEUGE

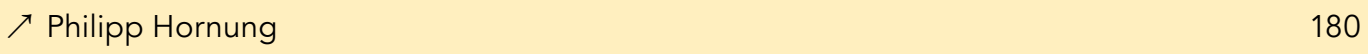

ROBOTIC FABRICATION AT THE ANGEWANDTE ROBOTICS LAB (ARL)

ROBOTER-FERTIGUNG IM ANGEWANDTE ROBOTICS LAB (ARL)

JOINTS / VERBINDUNGEN 190

\section{[3.0] REFLECTIONS AND SPECULATIONS / 201}

\section{MATERIALITY AND ITS AESTHETICS}

DIE MATERIALITÄT UND IHRE ÄSTHETIK

$\nearrow$ Karolin Schmidbaur

CONNECTION - EMPATHIC DIALOGUE

VERBINDUNG - EIN EMPATHISCHER DIALOG

$\nearrow$ Hélène Frichot

FOLLOW THE MATERIAL!

FOLGE DEM MATERIAL!

[4.0] A COMPLEMENTARY APPROACH

ERGÄNZENDER ANSATZ
$\nearrow$ Ferdinand Ludwig JOINING LIVING WOOD
LEBENDES HOLZ VERBINDEN

[5.0] APPENDIX 